\title{
Seismic Signal Discrimination between Earthquakes and Quarry Blasts Using Fuzzy Logic Approach
}

\author{
El Hassan Ait Laasri, Es-Saïd Akhouayri, Dris Agliz, and Abderrahman Atmani
}

Seismic Signal Processing Team, Electronic,

Signal Processing and Physical Modelling Laboratory,

Physics' Department, Faculty of Sciences,

IBN ZOHR University, B.P. 8106, Agadir, Morocco

\{hassan.or,driss_agliz,atmani_abderrahman\}@hotmail.com,

akhouayri@msn.com

\begin{abstract}
Seismic analysts identify earthquakes signals from those of explosions by visual inspection and calculating some characteristics of seismogram. Such work supposes a great deal of workload for seismic analysts. Therefore, an automatic classification tool reduces dramatically this arduous task, turns classification reliable, removes errors associated to tedious evaluations and changing of personnel. In this present paper we are interested in transforming the analysts' knowledge of classifying seismograms into an automated soft classification system based on fuzzy logic. This is primarily due to its capability of modelling human reasoning and decision-making, managing complexity and controlling computational cost. These capabilities are essential for manipulating high dimensionality and complexity of seismic signal. To conduct effective discrimination, relevant seismogram characteristics are extracted based on human experience. Using these characteristics, a fuzzy classifier is built and tested with real seismic data. The results are found to be encouraging.
\end{abstract}

Keywords: Seismic classification, Seismic signal processing, Fuzzy logic.

\section{Introduction}

The seismic database of Agadir was implemented in May 1998 by realization of an automatic station for detecting seismic events, and then in November 2001 by installation of the local seismic network [1]. The seismic events are detected by a power detector whereby the power over a short time-window (short-term average, STA) is compared with the power over a long time-window (long-term average, LTA). The basic idea of the algorithm is that an event is considered detected when the STA/LTA ratio exceeds a pre-determined threshold [2]. Because of several quarries located surrounding the Agadir city, many quarry explosions seismogram are recorded per day. As recorded explosions can mislead scientists interpreting the active tectonics and lead to erroneous results in the analysis of seismic hazards in the area, an event classification task is an important step in seismic signal processing. Such 
task analyses data in order to find to which class each recorded event belongs. In this work, we focus our efforts on identifying earthquakes seismograms from those of explosions in order to construct an accurate and classified database. In view of very high volume of data, the Considerable operations conducted by humans can be tedious and stressful. Therefore, constructing a reliable automatic discrimination system is a crucial issue. Many different seismogram discrimination methods have been developed, where the event signal is reduced to a set of features. The latter can be extracted from time-domain representation of the signal, time frequency representation or spectral representation. Spectral ratios $\mathrm{P} / \mathrm{S}$ and $\mathrm{P} / \mathrm{L}$ are commonly presented as good discriminants between earthquakes and quarry blasts [3] [4] [5] [6]. Nevertheless, due to the low magnitude and overlapped P and S waves of quarry blast events, we cannot use the previous discriminant methods.

As the seismic analyst arrived to distinguish between earthquake and explosion by visual inspection and calculating some characteristics of event signal, we are interested in transforming the analyst knowledge of classifying seismograms into an automated soft classifier. Such classifier can imitate the reasoning processes of experts in solving classification problem. The discriminant method was developed using fuzzy logic, considering that the latter is shown to be very useful in acquiring knowledge from human and a powerful tool to incorporate imprecise knowledge.

The rest of this article is organized as follow. Data characteristics are discussed in Section 2. Section 3 is devoted to the proposed discrimination method of fuzzy logic approach. Experimental results are shown in Section 4. Finally, Section 5 outlines some conclusions.

\section{Data}

The term "seismic source" is a comprehensive name for all events or, more generally, for any radiator of seismic waves. A seismic source can be described by its strength and its spatial and temporal characteristics, i. e., by parameters such as source dimension, geometry and time function of the radiation. Explosion sources are instantaneous and produce a spherically expanding compressional first motion in all directions while the tectonic earthquake sources produce first motions of different amplitude and polarity in different directions. Due to these source characteristics, it is obvious that the forces acting at the source of an explosion are very different from those acting at the source of a natural earthquake. These different forces introduce diverse characteristics to the seismic signal that can be readily used to identify each type of source process and discriminate between explosions and tectonic earthquakes.

In this section we present a data set of quarry blast events recorded by the seismic local network of Agadir. Natural earthquake seismograms are also collected for comparison study between these two events. Such study is useful to extract some characteristics of the signal generated by each type of these events. Fig. 1 depicts the vertical components seismogram of two earthquakes $(a, b)$ and two quarry blasts (c, d). 

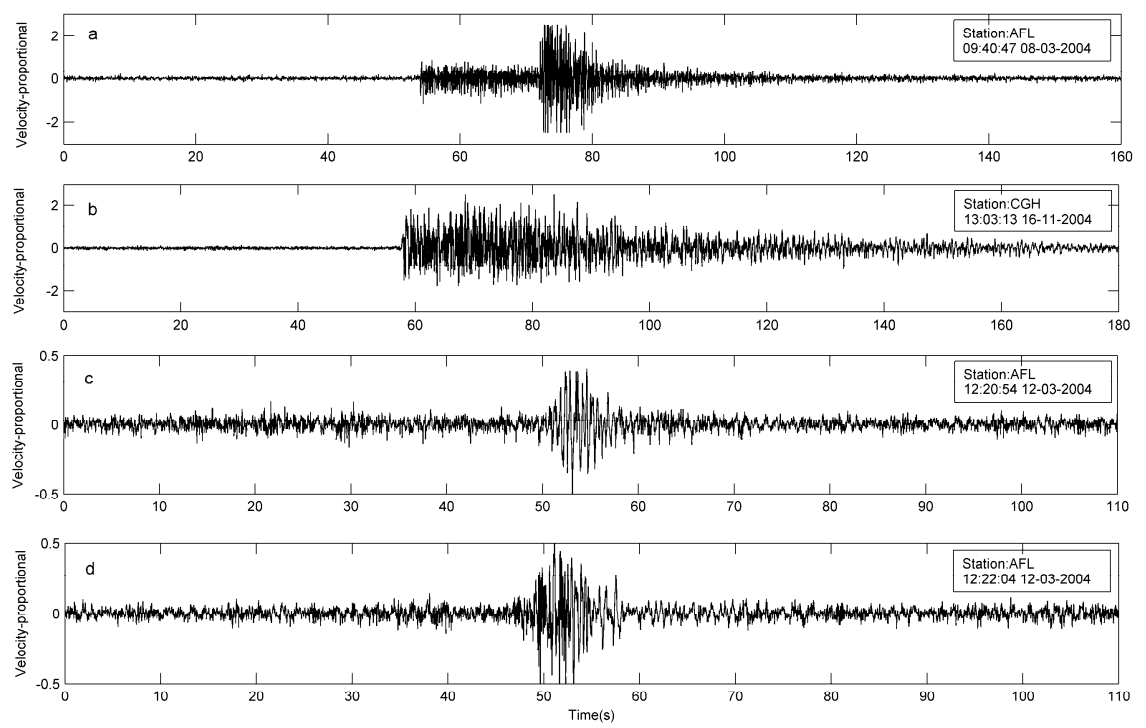

Fig. 1. Shows the vertical component seismogram of two earthquakes (a, b) and two quarry blasts $(\mathrm{c}, \mathrm{d})$ recorded by the local seismic network of Agadir

In a preliminary look at these seismograms, it seems that signal envelope is a promising discrimination parameter. The quarry blasts recording is characterized by overlapped $\mathrm{P}$ and $\mathrm{S}$ waves, less impulsive onset and short duration of coda waves. All these characteristics blend together to form a Gaussian envelope. Signal associated with an earthquake differs appreciably from that of explosion in that it involves large $\mathrm{S}$ waves, isolated or overlapped $\mathrm{P}$ and $\mathrm{S}$ waves (it depends on source-station distance) and exponential decay of coda amplitude with time.

Analysis of many explosion signals shows that all the events have almost the same envelope, and can be recognized using only the envelope. Unfortunately, not only the explosion signals show this feature; some earthquake signals have also the same envelope as explosion. In order to find another parameter which can differentiate these types of event, we analysed their signals in the frequency domain. In fig. 2, seismogram of an earthquake and a quarry blast with their associated FFT are compared. As can be seen in fig. 2, envelopes of these two events are quite similar, but the difference between their spectral content is clearly visible. It was observed that seismograms of quarry blast exhibit very low frequency amplitude below $1 \mathrm{~Hz}$. Contrary to earthquake seismograms, which often show very high frequency amplitude in the same band.

Another important parameter to be considered is time duration of the event. Time duration is influenced by many factors, mainly the characteristic dimensions of the source. Thus, it should be expected that time duration of natural earthquakes would be longer than the time duration of explosions. 

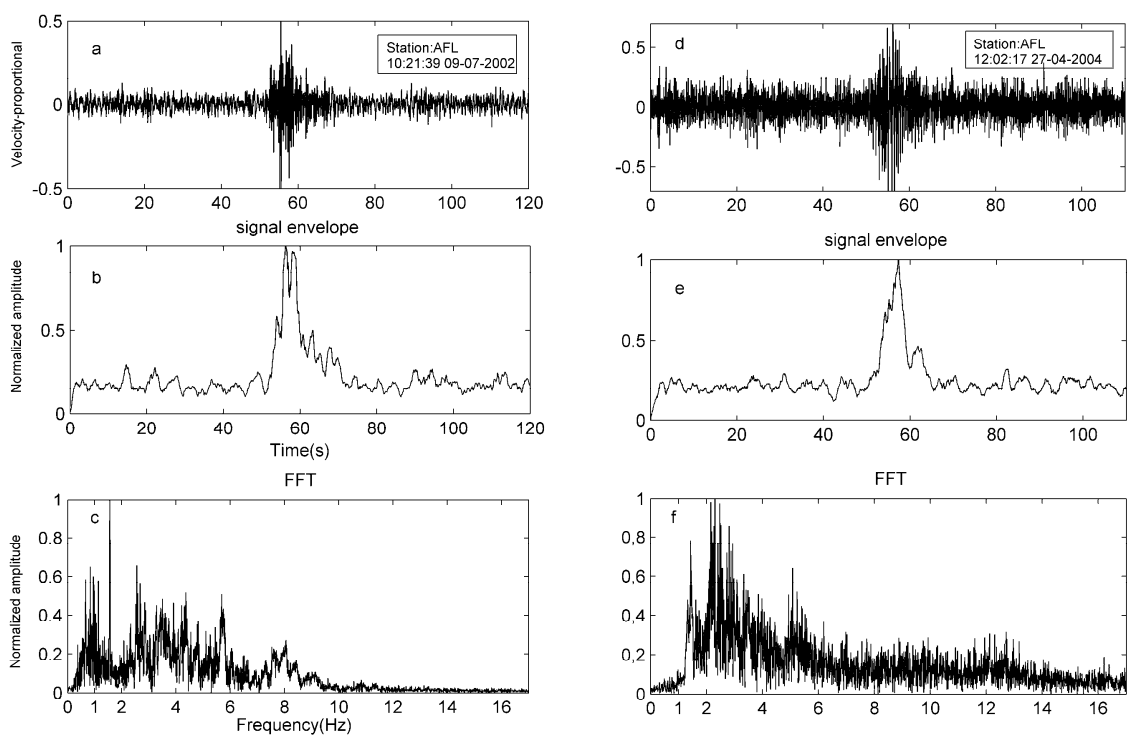

Fig. 2. Comparison between seismogram of an earthquake (a) and a quarry blast (d) and their corresponding envelope (b, e) and FFT (c, f)

Examination of the data displays that explosion records have durations of less than 40 seconds while tectonic earthquake records may last for several minutes.

The simplest variable which can be used for discrimination between earthquake and explosion is the hour of detection. This variable relay on the fact that explosion are exploded during specified hours and forbidden during the night. Therefore, we cannot record a quarry blast event during night time. Such variable is unreliable because it cannot separate seismograms recorded during day time, but can be reliable if it is used with other parameters.

\section{Method}

The problem of discrimination between earthquakes and quarry blasts is formulated as a problem in fuzzy logic. Such problem may be separated into two stages, feature extraction and classification.

\subsection{Feature Extraction}

The performance of classification method is affected by feature set used to reduce the high dimensiality of seismogram. Therefore, extract the most relevant feature from known seismograms contribute to more reliable discrimination of unknown seismograms. In this work the feature set defined by analysts corresponded to: 
Envelope: To extract the signal envelope, we use the Hilbert Transform which is capable of tracking the amplitude envelope of the signal:

$$
\begin{aligned}
H[x(t)] & =x(t) * \frac{1}{\pi t} \\
& =\frac{1}{\pi}\left[\int_{-\infty}^{+\infty} x(t) \cdot \frac{1}{(t-\tau)} \cdot d \tau\right]
\end{aligned}
$$

From the given signal $\mathrm{x}(\mathrm{t})$, a complex signal $\mathrm{A}[\mathrm{x}(\mathrm{t})]$ (also known as analytical signal) that is associated with the original signal can be constructed as :

$$
A[x(t)]=x(t)+j H[x(t)]
$$

The envelope of the signal is defined as:

$$
E(t)=\sqrt{x(t)^{2}+H[x(t)]^{2}}
$$

A finite impulse response filter (FIR) is designed to minimize the rapid variation of the envelope. As quarry blasts have usually the same envelope, we have chosen an envelope of this event as template envelope, which will be compared with upcoming events envelope.

Time Duration: The time duration td is defined as the total duration in seconds of the event record from the $\mathrm{P}$ wave onset tp to the end of the signal tend. The latter is defined as the point where the signal is no longer seen above the noise.

$$
t_{d}=t_{\text {end }}-t_{p}
$$

Hour: Quarry blast events occur during the time day from 11:00 a.m. to 02:00 p.m. and from 05:00 p.m. to 06:00 p.m. Beyond this time intervals the explosion are absent, and the seismicity pattern during these hours should not be affected by these events.

$$
\text { Hour }=\text { hour }+ \text { minute } / 60+\text { second } / 3600
$$

Frequency Content: The frequency amplitude of seismic event signal is calculated in the frequency band $[\mathrm{f} 1 \mathrm{f} 2]=[0.51] \mathrm{Hz}$.

$$
E_{s}=\int_{f_{1}}^{f_{2}} a(f) d f
$$

\subsection{Fuzzy Classifier}

Fuzzy logic theory, introduced by Zadeh [1965], deals with reasoning that is approximate rather than fixed and exact, which is the case in traditional logic theory. Fuzzy logic has been extended to handle the concept of partial truth, where the truth value 
may range between completely true and completely false. In classical concept of a set, the transition from one set to another is always abrupt; the membership of elements in a set is assessed in binary terms. So, an element either belongs or does not belong to the set. By contrast, the boundary of a fuzzy set is not precise. That is, the change from one class to its neighbours in a fuzzy set may be gradual rather than abrupt. This gradual change is expressed by a membership function valued in the real unit interval $[0,1]$. Because the real world data, where information is often incomplete or sometimes unreliable, do not have sharply defined boundaries, fuzzy classifier provides the appropriate tool to manipulate the real data. What makes Fuzzy Logic so powerful is its ability of describing a system in linguistic terms rather than in terms of numbers. This enables the design of such system with more human-like reasoning using linguistic terms of natural languages.

Let $\mathrm{C}=(\mathrm{C} 1, \mathrm{C} 2)$ be the two classes of seismic event, which can be described by a set of features or attributes $\mathrm{Xi}, \mathrm{i}=1, \ldots$, n. i.e., a given event to classify is an element $\mathrm{x}=(\mathrm{x} 1, \ldots, \mathrm{xn})$ of $\mathrm{X} 1 \times \ldots \times \mathrm{Xn}$, where $\mathrm{xi}$ is the value taken by attribute $\mathrm{i}$ for this event. In the sequel, Xi will indicate either the attribute (or variable) itself or its set of values, while xi indicate possible values of $\mathrm{Xi}$. The problem of designing the classifier is to define a mapping $F$ such as:

$$
\mathrm{F}: \mathrm{X} 1 \times \mathrm{X} 2 \times \ldots \times \mathrm{Xn} \rightarrow \mathrm{C}
$$

By employing fuzzy logic as a nonlinear mapping $\mathrm{F}$, it is possible to describe the degree to which an event belongs to one class or the other.

In order to solve a fuzzy classification problem, it is necessary to fuzzify inputs (Fuzzification), determine all IF-THEN rules (rule base), process them (Interference engine) and provide result in a usable and understandable form (Defuzzification) [7][8].

Fuzzification: Fuzzification interface converts inputs of system into fuzzy variable. It contains predefined sets of linguistic terms and the degree to which inputs belong to each of the appropriate fuzzy sets is determined via membership functions. Qualitative interpretation of different available values of input variables is achieved by fuzzification.

Fuzzy Rules: The rules refer to variables and the adjectives that describe those variables. Rules are fuzzy conditional statements (implications) and usually expressed in the form:

\section{IF variable IS adjective THEN class}

Fuzzy Inference: Fuzzy inference simulates human decision making to assign a class to each input based on his knowledge and interpretation of the input vector. So, the point of fuzzy inference is to map an input space to an output space, and the primary mechanism for doing this is the list of 'if-then' rules. All rules are evaluated in parallel, and the order of the rules is unimportant. The most commonly types of fuzzy inference systems that can be implemented in Fuzzy Logic are: Mamdani-type and Sugeno-type which is used in this paper [9]. In fact they are similar in many respects. The main difference between them is that the output membership functions are only 
linear or constant for Sugeno-type fuzzy inference. A typical rule in a Sugeno fuzzy model has the following form:

If Input $1=\mathrm{x}$ and Input $2=\mathrm{y}$, then Output $\mathrm{z}$ is:

$$
\mathrm{z}=\mathrm{ax}+\mathrm{by}+\mathrm{c}
$$

where $\mathrm{a}, \mathrm{b}$ and $\mathrm{c}$ are constants.

Defuzzification: Defuzzification interface converts fuzzy outputs into numerical value, which corresponds to event class.

\section{$4 \quad$ Results and Discussion}

The dataset contains two classes of seismic signals: earthquake and quarry blast. Discriminative parameters are illustrated in Table 1, where their corresponding fundamental descriptive statistical information for each class is provided. Such information is useful in defining the membership function. To represent the variable Hour, the histogram was used (fig. 3).

Table 1. Discriminative parameters and their corresponding fundamental descriptive statistical information for the two classes

\begin{tabular}{lrrrrrr}
\hline & \multicolumn{2}{c}{ Duration } & \multicolumn{2}{c}{ envelope } & \multicolumn{2}{c}{ frequency } \\
\hline & earthquake & $\begin{array}{c}\text { Quarry } \\
\text { blast }\end{array}$ & earthquake & $\begin{array}{l}\text { Quarry } \\
\text { blast }\end{array}$ & earthquake & $\begin{array}{l}\text { Quarry } \\
\text { blast }\end{array}$ \\
\hline minimum & 13.69 & 9.06 & 0.47 & 0.09 & 0.08 & 0.22 \\
maximum & 736.80 & 32.03 & 0.98 & 0.55 & 190.12 & 7.44 \\
mean & 135.39 & 17.30 & 0.82 & 0.39 & 20.51 & 2.38 \\
Standard & 150.45 & 4.83 & 0.12 & 0.09 & 39.51 & 1.58 \\
deviation & & & & & & \\
\hline
\end{tabular}
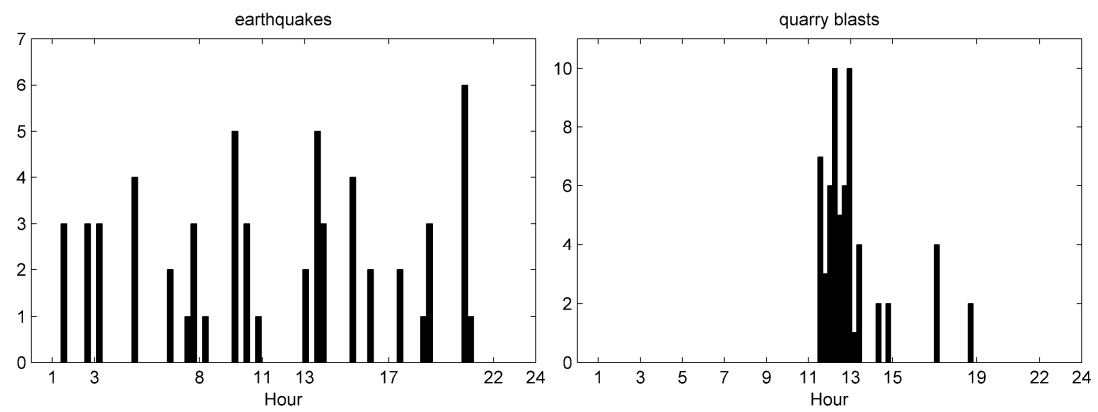

Fig. 3. Histogram of the parameter Hour for both earthquake class and quarry blast one

From these statistical data, it turns out that there is no single parameter with fixed classification threshold; as it can be seen in Table 1, similar values can be found for each parameter in the two classes. Therefore a single parameter cannot distinguish 
between the two classes because they are overlapped. The most accepted solution for discrimination is the combination of these different parameters. At this point, the fuzzy inference system plays an important role. Fuzzy classes reflect reality better and allow decision makers to describe input attributes and output classes more intuitively using linguistic variables, overlapping classes and approximate reasoning. Events that belong to more than one class are treated in all classes where they have partial membership.

The classification system is made up using the first-order Sugeno fuzzy model with fore inputs and an output. Trapezoidal type membership function is chosen to be more suitable for the input variable because of its shape. Based on descriptions of the input (Hour is night, envelope is Gaussian, duration is short, frequency is low...etc) and output classes (earthquake, quarry blast), 09 rules are constructed. With 120 events (60 earthquakes, 60 quarry blasts), results were as following:

- correctly classified events: 120

- misclassified: 0

- accuracy: $100 \%$

The results of this research have shown that the fuzzy approach is perfectly suitable for distinguishing earthquake events from quarry blast ones. Such technique, achieved a high discrimination performance with low complexity, could be employed in real time discrimination. Moreover, by using fuzzy logic rules, the maintenance of the structure of the algorithm decouples along fairly clean lines. The features characteristics of each class might change in the future, but the underlying fuzzy classifier will be the same. For example, exploded hour of quarry blasts can be changed in the future but, the system can be recalibrated quickly by simply shifting the fuzzy set that defines Hour or just rewriting the fuzzy rules without touching the complex programming code. Also, adding more rules to the bottom of the list to increment or expand the scope of the knowledge-base, as processes develop or new events are found, is relatively easy and without needing to undo what had already been done. In other words, the subsequent modification was pretty easy. The last statement is perhaps the most important one and deserves to be addressed here. Since fuzzy logic is built on top of linguistic terms used by ordinary people on a daily Basis, fuzzy logic allows anyone to edit and modify the rules without worrying about underlying code.

\section{Conclusion}

In this paper, an automated discriminant method between earthquakes and explosions in Agadir database is developed using fuzzy logic. Each event is represented by a set of features deduced from corresponding signal. The fuzzy system interprets the values in the input vector and, based on some set of rules, assigns each input to its class. Fuzzy logic is used due to its human-like-reasoning nature, its feasibility of implementation nonlinear problems and its capabilities to deal with uncertainties and imprecision, but otherwise the fuzzy should be considered in view of its simplicity and transparency. This simplicity however does not limit its effectiveness. In this work fuzzy classifier appear as a 
powerful tool to deal with seismic signal, which is distorted, weakly, noisy and complex. Fuzzy classifier results show good performance with low complexity. However, fuzzy methods are still dependent on expert knowledge.

\section{References}

1. Akhouayri, E., Ait Laasri, H., Agliz, D., Atmani, A.: Agadir's seismic central acquisition management. In: 27th European Center for Geodynamics and Seismology, ECGS, Luxembourg, Nouvember 17-19 (2008)

2. Akhouayri, E., Agliz, D., Fadel, M., Ait Ouahman, A.: Automatic detection and indexation of seismic events. AMSE Periodicals, Advances in Modeling and Analysis, série C 56(3), 59-67 (2001)

3. Yildırıma, E., Gulbag, A., Horasana, G., Dogan, E.: Discrimination of quarry blasts and earthquakes in the vicinity of Istanbul using soft computing techniques. Computers \& Geosciences, 01-09 (2010)

4. Allmann, P.B., Shearer, P.M., Hauksson, E.: Spectral discrimination between quarry blasts and earthquakes in Southern California. Bulletin of the Seismological Society of America 98(4), 2073-2079 (2008)

5. Ursino, A., Langer, H., Scarfi, L., Grazia, G.D., Gresta, S.: Discrimination of quarry blasts from tectonic microearthquakes in the Hyblean Plateau (south-eastern Sicily). Annali di Geofisica 44, 703-722 (2001)

6. Orlic, N., Loncaric, S.: Earthquake-explosion discrimination using genetic algorithm-based boosting approach. Computers \& Geoscience 36, 179-185 (2010)

7. Ruiz Reyes, N., Vera Candeas, P., Garcia Galan, S., Munoz, J.E.: Two-stage cascaded classification approach based on genetic fuzzy learning for speech/music discrimination. Engineering Applications of Artificial Intelligence 23, 151-159 (2010)

8. Kovacic, Z., Bogdan, S.: Fuzzy Controller Design -Theory and Applications. Taylor \& Francis Group, LLC, Boca Raton (2006)

9. Sivanandam, S.N., Sumathi, S., Deepa, S.N.: Introduction to Fuzzy Logic using MATLAB. Springer, Heidelberg (2007) 